\title{
Feasibility of Improved Attenuation Correction for SPECT Reconstruction in the Presence of Dense Materials Using Dual-Energy Virtual Monochromatic CT: A Phantom Study
}

\author{
Sachiko Yamada1,2*, Takashi Ueguchi33,4, Eku Shimosegawa5, Koichi Fujino', \\ Takeshi Shimazu6, Kenya Murase $^{2}$, Jun Hatazawa ${ }^{7}$ \\ ${ }^{1}$ Department of Radiology, Osaka University Hospital, Osaka, Japan \\ ${ }^{2}$ Department of Medical Physics and Engineering, Osaka University Graduate School of Medicine, Osaka, Japan \\ ${ }^{3}$ Center for Information and Neural Networks, National Institute of Information and Communications \\ Technology, Osaka, Japan \\ ${ }^{4}$ Graduate School of Frontier Biosciences, Osaka University, Osaka, Japan \\ ${ }^{5}$ Department of Molecular Imaging in Medicine, Osaka University Graduate School of Medicine, Osaka, Japan \\ ${ }^{6}$ Department of Traumatology and Acute Critical Medicine, Osaka University Graduate School of Medicine, \\ Osaka, Japan \\ ${ }^{7}$ Department of Nuclear Medicine and Tracer Kinetics, Osaka University Graduate School of Medicine, Osaka, \\ Japan \\ Email: *yamada@hp-rad.med.osaka-u.ac.jp
}

Received 30 September 2015; accepted 1 December 2015; published 4 December 2015

Copyright (C) 2015 by authors and Scientific Research Publishing Inc.

This work is licensed under the Creative Commons Attribution International License (CC BY).

http://creativecommons.org/licenses/by/4.0/

(c) (i) Open Access

\section{Abstract}

Objective: Computed tomography (CT)-based attenuation correction (CTAC) offers the clear benefit of reliable reconstruction of single-photon emission computed tomography (SPECT) images through its ability to achieve object-specific attenuation maps, but artifacts from dense materials often deteriorate CTAC performance. Therefore, we investigate the feasibility of CTAC in the presence of dense materials using dual-energy virtual monochromatic CT data. Methods: A sodium pertechnetate-filled cylindrical uniform phantom, with a pair of undiluted iodine syringes attached, is scanned with a dual-source CT scanner to obtain both single-energy $(120 \mathrm{kVp})$ polychromatic and dual-energy ( $80 \mathrm{kVp} / 140 \mathrm{kVp}$ with tin filtering) virtual monochromatic CT images. The single-energy and the dual-energy CT images are then converted to attenuation maps at 141

\footnotetext{
"Corresponding author.
} 
keV. SPECT images are reconstructed from ${ }^{99 \mathrm{~m} T c}$ emission data of the phantom using each single-energy and dual-energy attenuation map and incorporating CTAC procedure. A region-of-interest analysis is performed to quantitatively compare the attenuation maps between the single-energy and the dual-energy techniques, each at an iodine-free position and a position adjacent to the iodine solutions. Results: At the iodine-free position, the phantom provides a uniform distribution of attenuation maps in both the single-energy and the dual-energy techniques. In the presence of adjacent iodine solutions, however, severe artifacts appeare in the single-energy CT images. These artifacts make attenuation values fluctuate, resulting in erroneous pixel values in the CTAC SPECT images. In contrast, dual-energy CT strongly suppresses the artifacts and hence improves the uniformity of the attenuation maps and the resultant SPECT images. Conclusions: Dual-energy CT with virtual monochromatic reconstruction has the potential to substantially reduce artifacts arising from dense materials. It has the potential to improve the accuracy of attenuation maps and the resultant CTAC SPECT images.

\section{Keywords}

\section{SPECT, Attenuation Correction, Dual-Energy CT, Virtual Monochromatic Imaging, Artifact} Reduction

\section{Introduction}

Reliable quantification of tracer distributions in single-photon emission computed tomography (SPECT) requires accurate attenuation correction (AC) of emission data, which entails an accurate determination of the attenuation map of the object [1]-[3]. Compared to the conventional Chang attenuation correction method [4], x-ray computed tomography-based attenuation correction (CTAC) offers a clear benefit through its ability to correct non-uniform attenuation of photons inside the object [5] [6]. While CTAC first became a well-established clinical application in positron emission tomography (PET) combined with CT, CTAC itself was first introduced with regard to SPECT in the early 1990s through the development of a combined SPECT and CT prototype system for small animals [7]-[12]. The concept of CTAC using separately acquired CT images that was also reported in the late 1980s [13]. The advantage of the low-noise and high-throughput performance of CTAC over traditional transmission scans has been well recognized in clinical PET [14]-[16]. These features have also attracted increasing interest in SPECT in recent years, particularly with the introduction of hybrid SPECT/CT scanners [6] [17] [18].

The reliability of CTAC depends critically on the accuracy of linear attenuation coefficient (LAC) values derived from CT images. CT images are, however, prone to a wide variety of artifacts from dense materials [19]. These artifacts are quite common in CT images of patients who have metallic implants, such as dental fillings, neurosurgical clips or coils, orthopedic prostheses, or cardiac pacemakers. The artifacts also result from intravenous or oral contrast materials. Several factors such as beam hardening have been reported as potential sources of the artifacts [19]-[21]; the artifacts cause CT numbers (and thus LAC values) to deviate from their true values regardless of their origins. The use of such corrupted CT data in the subsequent CTAC procedure can result in an erroneous estimation of tracer uptake in regions contaminated with the artifacts [22] [23].

One of the recently introduced CT techniques to mitigate artifacts from dense materials is dual-energy imaging. It permits the reconstruction of "virtual" monochromatic images, which can provide better image quality than the standard single-energy scan when dense materials exist in the scanning field of view [20] [24]-[27]. The strategy of dual-energy CT was first proposed in the late 1970s [24] [28], but only recently did it become available for routine use in clinical CT scanners. In this study, we investigate the feasibility of AC using dual-energy virtual monochromatic CT data. Although the use of dual-energy CT in AC has been reported in the literature [29]-[32], most of those reports are related to AC with PET/CT, focusing on the energy scaling strategy, i.e. how to generate attenuation maps at $511 \mathrm{keV}$ from the CT data. In contrast, we focus on the ability to reduce artifacts arising from dense materials in order to provide more accurate attenuation maps for SPECT reconstruction. 


\section{Materials and Methods}

\subsection{Phantom}

This study was performed with a cylindrical acrylic phantom (16 cm in diameter and $33 \mathrm{~cm}$ in height) and two syringes of undiluted iodine contrast material (Iopamidol 370, Bayer, Osaka, Japan; total amount of $100 \mathrm{ml}$ per syringe, with an iodine concentration of $370 \mathrm{mg} / \mathrm{ml}$ ). The cylindrical phantom was homogeneously filled with ${ }^{99 \mathrm{~m}}$ Tc sodium pertechnetate having a specific activity of $42 \mathrm{MBq} / \mathrm{L}$. Figure 1 shows a radiograph of the phantom (CT localizer, anterior view). The two iodine syringes were mounted adjacent to, but some distance apart from, the cylindrical phantom. This phantom setup was intended to produce severe artifacts in the CT images, allowing us to investigate their effects on the attenuation maps.

\subsection{CT Acquisition}

Both single-energy and dual-energy CT scans for AC were performed with a dual-source CT scanner (SOMATOM Definition Flash, Siemens, Erlangen, Germany). This scanner utilizes two distinct source-detector systems arranged at an angle separation of $95^{\circ}$, enabling simultaneous acquisition of both high- and low-energy CT images with different tube voltages, currents, and additional filters. The scanner was operated in helical mode for both scans. The phantom was positioned on the patient table of the scanner so that the longitudinal axis of the phantom was parallel to that of the table. For the single-energy images, the scans were acquired using one $\mathrm{x}$-ray tube operating at $120 \mathrm{kVp}$ and $50 \mathrm{mAs}$. For the dual-energy scan, the scanner was operated with one x-ray tube at $140 \mathrm{kVp}$ and $19 \mathrm{mAs}$, and the other at $80 \mathrm{kVp}$ and $50 \mathrm{mAs}$. An additional tin filter was used for the $140 \mathrm{kVp}$ beam to improve spectral separation and keep the radiation dose similar to, or lower than, the single-energy CT [33] [34]. The volume CT dose index (CTDIvol) for the dual-energy scan was $1.93 \mathrm{mGy}$, while that for the single-energy scan was $3.43 \mathrm{mGy}$. The slice thickness and the reconstructed field-of-view were $3 \mathrm{~mm}$ and $350 \mathrm{~mm}$, respectively, for both the scans. The images were reconstructed with standard body kernels (B30f for singleenergy and D26f for dual energy scans). For the dual-energy scan, the virtual monochromatic images were synthesized using dedicated software (syngo, Dual Energy, "Monoenergetic", Siemens), which allowed the interactive reconstruction of monochromatic images at arbitrary energies throughout a wide range, from 40 to $190 \mathrm{keV}$ in 1-keV increments. Although dual-energy CT can synthesize virtual monochromatic images at the photon energies of the radionuclide, i.e. $141 \mathrm{keV}$ for technetium-99 m, the efficacy of artifact reduction is not necessarily maximal at that energy [25] [27]. We thus reconstructed virtual monochromatic images at energy of $110 \mathrm{keV}$, which visually minimized the artifacts. This reconstructed energy was determined by a consensus of two of the authors, each of whom have more than 10 years of clinical experience in the field of CT.

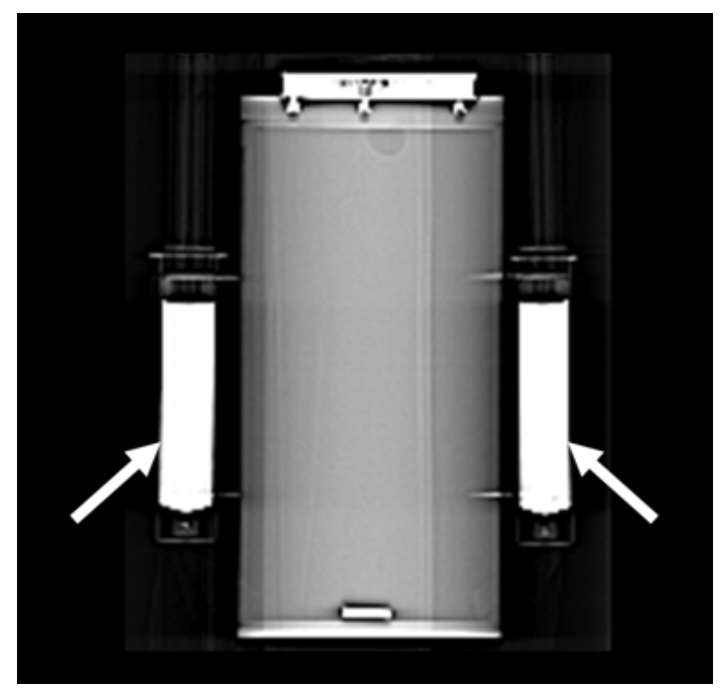

Figure 1. Localizer image (anterior view) of the cylindrical phantom used in this study. Two undiluted iodine syringes (arrows) were mounted beside the phantom in order to produce artifacts in the CT images. 


\subsection{Conversion of CT Number to LAC Value}

In order to generate attenuation maps of the imaged object, the CT images (both the single-energy and the dual-energy scans) were reformatted so that they had the same matrix format and same voxel dimensions as the SPECT data described below. Then their pixel values in Hounsfield Units (HU) were converted using HU-toLAC calibration curves to produce attenuation maps at $141 \mathrm{keV}$ in units of $\mathrm{cm}^{-1}$. The generation of the HU-toLAC calibration curves was accomplished by acquiring CT images of a calibration phantom (Gammex 467 tissue characterization phantom, Gammex Inc., Middleton, WI) containing 13 different materials of known composition (and thus of known LAC values at the given photon energy) from which the CT number (in HU) of each material could be measured (Figure 2). The LAC values of the 13 materials at $141 \mathrm{keV}$ ranged from 0.043 $\mathrm{cm}^{-1}$ (simulating lung tissue) to $0.280 \mathrm{~cm}^{-1}$ (simulating cortical bone). This procedure was performed for both the single-energy and the dual-energy CT scans, each with the same scan parameters described above. The CT number for each material was determined by measuring the mean $\mathrm{HU}$ values within a centered region of interest (ROI) inside the material. Piecewise linear HU-to-LAC calibration curves for both the single-energy and the dual-energy scans were then obtained by plotting known LAC values of the materials at $141 \mathrm{keV}$ against their measured HU values.

\subsection{SPECT Acquisition}

SPECT acquisition was performed using a dual-head gamma camera system (Symbia T6, Siemens) equipped with low-energy, high-resolution collimators. The ${ }^{99 \mathrm{~m}} \mathrm{Tc}$-filled phantom (along with the adjacent iodine syringes) was placed on the patient table of the scanner so that the central longitudinal axis of the phantom passed through the rotation axis of the gamma cameras. Emission data were collected in the step-and-shoot mode with two opposing detectors going through 45 steps, each separated by 4 degrees. An automatic body-contouring feature was used to obtain the optimal detector-to-phantom distance. Other acquisition parameters were as follows: acquisition matrix, $128 \times 128$; pixel size, $4.8 \mathrm{~mm}$; acquisition time, $30 \mathrm{~s}$ per projection; main energy window, $140 \pm$ $10 \mathrm{keV}$; lower energy window for scatter correction, $120 \pm 10 \mathrm{keV}$. SPECT images were reconstructed using the vendor-supplied ordered-subset expectation maximization iterative reconstruction algorithm (Flash 3D, Siemens) that incorporated CTAC as well as collimator-specific resolution recovery and a dual-energy-window scatter correction [35] [36]. An 8-mm Gaussian filter was applied to the projection data to facilitate noise reduction. The reconstruction was performed with a combination of 6 subsets and 9 iterations.

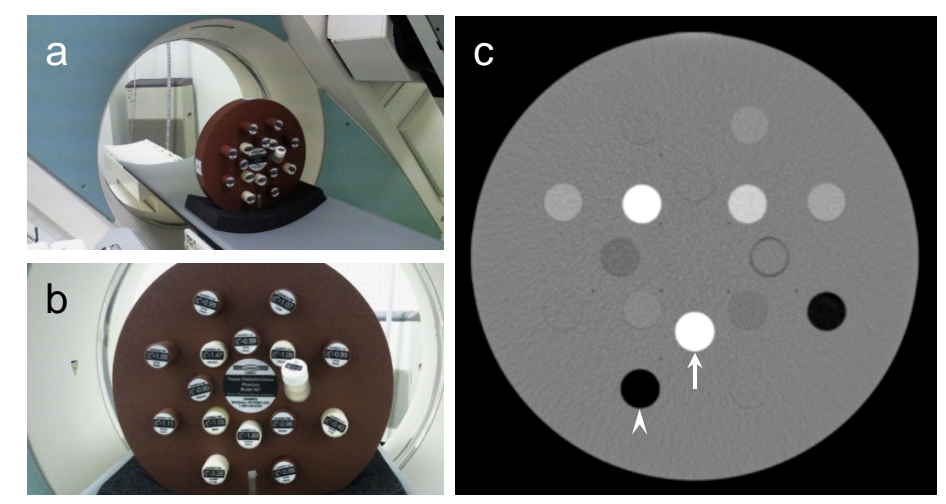

Figure 2. Photographs ((a) and (b)) and CT image (c) of the calibration phantom used to obtain the HU (Hounsfield units)-to-LAC (linear attenuation coefficients) conversion curves. The phantom base was made from "Solid Water", which has a physical density (PD) of $1.017 \mathrm{~g} / \mathrm{cm}^{3}$ and an electron density (ED) relative to water of 0.988 . Sixteen rods (four of which were also made from Solid Water and thus indistinguishable from the phantom base on the CT image) were inserted into the phantom base according to the manufacturer's instructions. The rods with the highest (arrow in (c)) and the lowest (arrowhead in (c)) densities on the CT image correspond to the cortical bone (PD, $1.824 \mathrm{~g} / \mathrm{cm}^{3}$; $\mathrm{ED}, 1.696)$ and the lung (PD, $0.29 \mathrm{~g} / \mathrm{cm}^{3}$; ED, 0.284), respectively. 


\subsection{Analysis}

The analysis focused on the accuracy of LAC values obtained from the uniform phantom in the presence of dense materials, i.e. the two iodine solutions, since LAC values are probably more susceptible to the presence of dense objects than are the pixel values of resultant SPECT images. For each single-energy and dual-energy CT scan, two transaxial attenuation maps were selected for the analysis: one acquired at the slice position passing through the center of the iodine solution (referred to as the "iodine position") and the other acquired at the position that did not pass through the iodine solution (referred to as the "iodine-free position"). The quantitative distribution of LAC values in terms of dispersion was evaluated by means of their first and third quartiles, 10th and 90th percentiles, as well as standard deviation. In order to obtain these statistics, we measured LAC values in a circular ROI with a diameter of 25 pixels, which encompassed approximately $75 \%$ of the interior diameter of the phantom; the circular ROI was positioned at the center of the phantom on each attenuation map. The spatial distribution of relative errors in the measured LAC values was also assessed by generating an "LAC error map" for each attenuation map. The relative error was defined as the percentage difference between measured and reference LAC values relative to the reference value, where the reference value was the theoretically expected LAC value of water at $141 \mathrm{keV}$, which was $0.1536 \mathrm{~cm}^{-1}$. The relative error was calculated for each pixel in an attenuation map, resulting in the LAC error map.

\section{Results}

HU-to-LAC conversion curves for both the single-energy and the dual-energy CT scans are shown in Figure 3. Data in each scan were fitted bilinearly with a flexion point of $0 \mathrm{HU}$.

Figure 4 shows single-energy CT images ((a) and (d)), their corresponding attenuation maps ((b) and (e)), and resultant CTAC SPECT images ((c) and (f)), acquired at the iodine-free position ((a)-(c)) and the iodine position ((d)-(f)). In the absence of the adjacent iodine solutions ((a)-(c)), each image depicts a uniform internal structure. In the presence of the iodine solutions, however, there were substantial dark and bright artifacts between them ((d) and (e)). These artifacts are much more prominent in the CT image (d) than in the attenuation map (e). The artifacts also appeared in the CTAC SPECT images (f).

Figure 5 shows dual-energy virtual monochromatic CT images ((a) and (d)), their corresponding attenuation maps ((b) and (e)), and resultant CTAC SPECT images ((c) and (f)), acquired at the iodine-free position ((a)-(c)) and the iodine position ((d)-(f)). In the absence of the adjacent iodine solutions, each image depicts a uniform internal structure ((a)-(c)). In the presence of iodine solutions, each image again shows almost uniform internal structure ((d)-(f)). In contrast to the single-energy images shown in Figure 4, the artifacts are substantially reduced in the dual-energy CT image and the corresponding attenuation map (Figure 5(d) and Figure 5(e)). The dual-energy CTAC SPECT image (Figure 5(f)) demonstrates improved uniformity compared with the singleenergy CTAC SPECT image (Figure 4(f)).

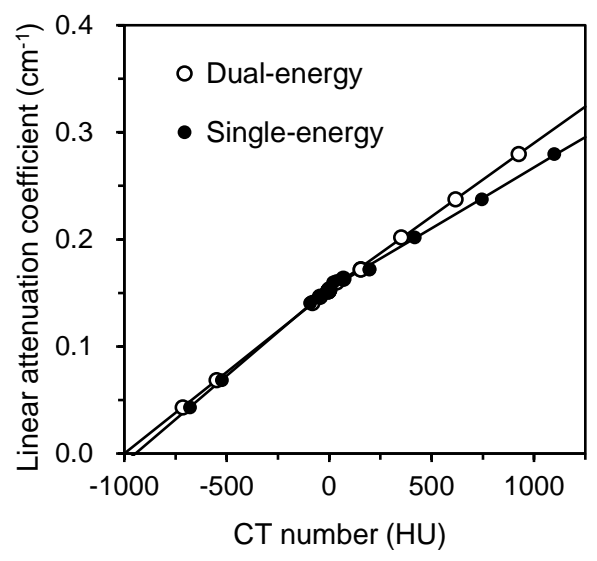

Figure 3. The HU-to-LAC conversion curves for the single-energy and the dual-energy CT data. The data in each scan were fitted bilinearly with a flexion point of $0 \mathrm{HU}$. 


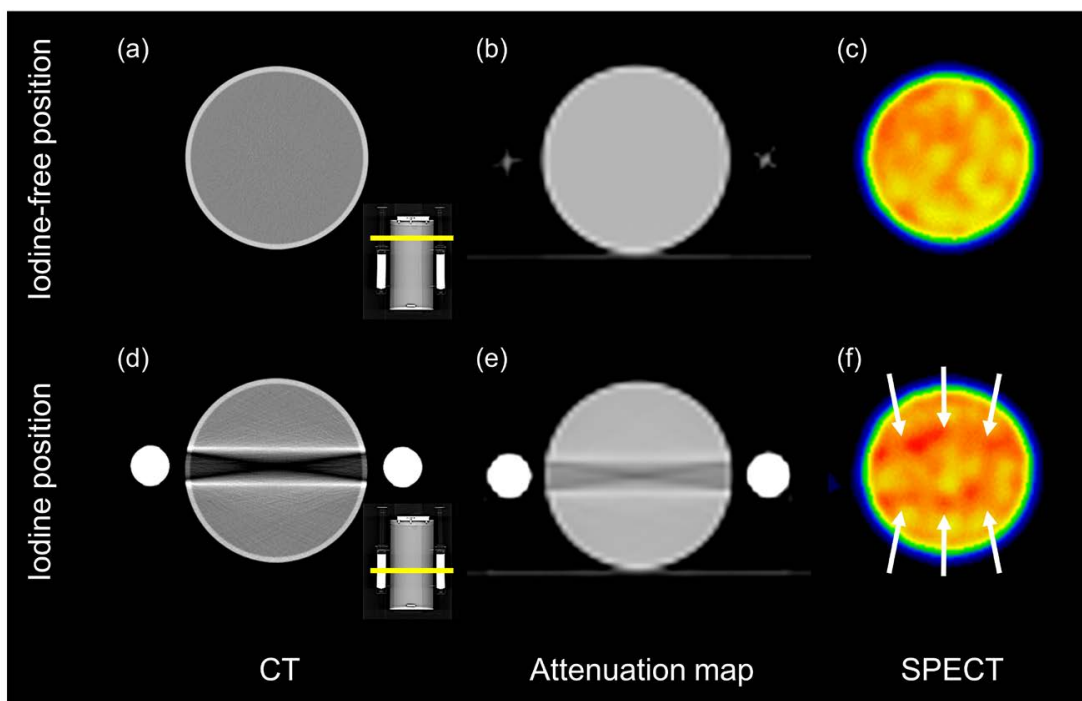

Figure 4. Conventional single-energy CT images ((a) and (d)), their corresponding attenuation maps ((b) and (e)), and CTAC SPECT images ((c) and (f)) scanned at the iodine-free position ((a)-(c)) and at the iodine position ((d)-(f)). For CT images, localizer images with the corresponding reference lines are also shown in the lower right corner. The iodine solutions cause severe artifacts in the CT image (d) and its corresponding attenuation map (e). Artifacts are also present in the CTAC SPECT image ((f), arrows).

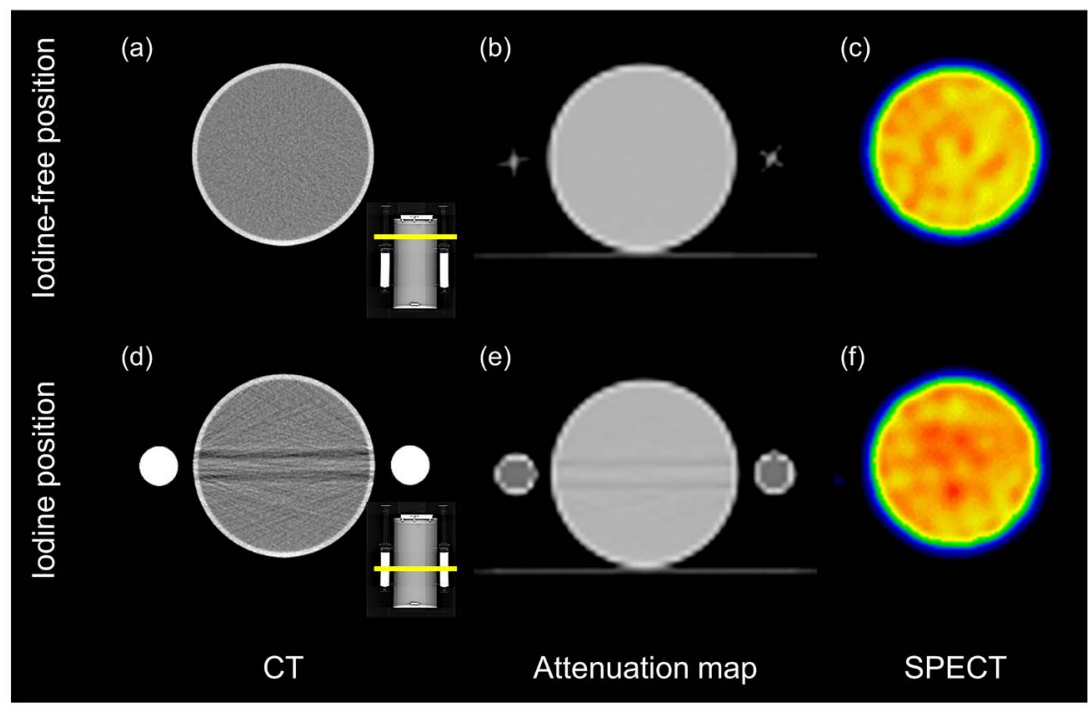

Figure 5. Dual-energy CT images ((a) and (d)), their corresponding attenuation maps ((b) and (e)), and CTAC SPECT images ((c) and (f)) scanned at the iodine-free position ((a)-(c)) and at the iodine position ((d)-(f)). For CT images, localizer images with the corresponding reference lines are also shown in the lower right corner. The artifacts arising from iodine solutions are substantially reduced in the CT image (d) and the attenuation map (e), providing better uniformity in the CTAC SPECT image (f).

Figure 6 shows a box-and-whisker plot for the measured LAC values, where the ends of the whisker represent the 10th and 90th percentiles. At the iodine-free position, both the single-energy and the dual-energy techniques provided LAC values in the 10th-to-90th percentile range of less than $0.001 \mathrm{~cm}^{-1}$, which means that the distribution of the values were quite uniform. At the iodine position, however, the single-energy technique yielded values of $0.035 \mathrm{~cm}^{-1}$ and $0.005 \mathrm{~cm}^{-1}$ for the 10th-to-90th percentile and the interquartile ranges, respectively, 


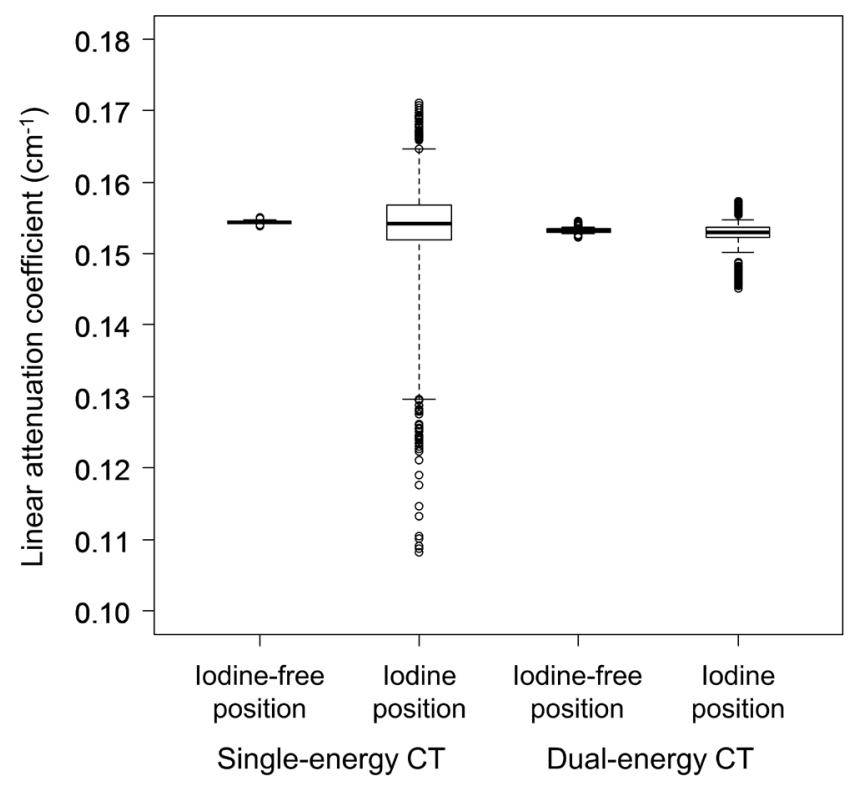

Figure 6. Box-and-whisker plot of the LAC values derived from both the single-energy and the dual-energy CT data. Both ends of the whisker represent 10th and 90th percentiles. Outliers are plotted with open circles. At the iodine-free position, the 10th-to-90th percentile ranges for both the single-energy and the dual-energy techniques are quite small. At the iodine position, however, the LAC values derived from the single-energy CT exhibit much greater variation than those from the dual-energy CT.

indicating that the distribution of the measured LAC values strongly fluctuated due to the artifacts. The use of the dual-energy technique substantially shortened these statistics at the iodine position; the 10th-to-90th percentile and the interquartile ranges were $0.005 \mathrm{~cm}^{-1}$ and $0.001 \mathrm{~cm}^{-1}$, respectively, indicating that the uniformity of the LAC values was substantially improved. The standard deviations of the measured LAC values at the iodine-free and the iodine positions were $<0.001 \mathrm{~cm}^{-1}$ and $0.013 \mathrm{~cm}^{-1}$, respectively, with the single-energy technique, and were $<0.001 \mathrm{~cm}^{-1}$ and $0.002 \mathrm{~cm}^{-1}$, respectively, with the dual-energy technique.

LAC error maps for both the single-energy and the dual-energy techniques are shown in Figure 7. At the iodine-free position, relative errors were almost $0 \%$ for the entire region inside the phantom ((a) and (b)). At the iodine position, however, the single-energy CT resulted in substantial positive and negative error values at the region between the two iodine solutions (c). The positive error values corresponded to the bright artifacts in the CT image, while the negative error values corresponded to the dark artifacts. The use of dual-energy CT substantially decreased those errors (d). It should be noted that since these LAC error maps were calculated relative to the theoretical LAC value of water, only error values inside the phantom are meaningful.

\section{Discussion}

In this study, we performed a simple phantom experiment to investigate if dual-energy virtual monochromatic CT could provide better attenuation maps than conventional single-energy CT in the presence of dense materials. Two syringes filled with undiluted iodine contrast material were used as extremely dense materials [23]. The distributions of the pixel values on the attenuation maps were compared between two positions: one in the presence of the iodine solution and the other in its absence. To avoid subjectivity, we did not perform any visual assessment. As expected, severe artifacts arising from the iodine solutions appeared in the single-energy CT image, resulting in an erroneous attenuation map. Substantial errors were distributed between the two iodine solutions but were not found in the absence of iodine. Some of these artifacts, which had positive error values propagated into the CTAC SPECT image. In contrast, artifacts were almost invisible in the dual energy CTAC SPECT image, although small remnants of the artifacts could still be seen on both the virtual monochromatic CT image 

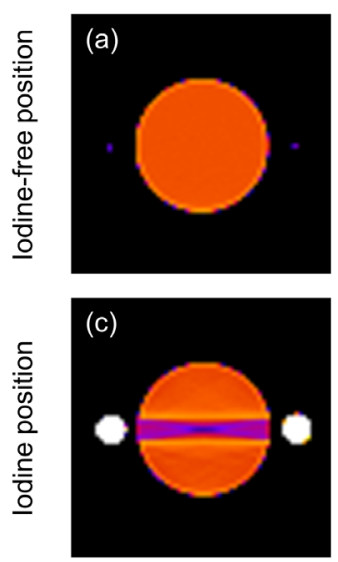

Single-energy CT

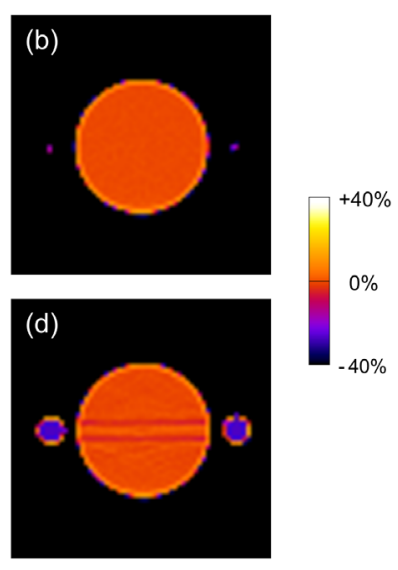

Dual-energy CT

Figure 7. LAC error maps showing spatial distributions of error values for LAC relative to LAC of water at $141 \mathrm{keV}$. At the iodine position, substantial error values are observed at the region between the two iodine solutions when the single-energy technique is used (c). This error was substantially reduced by the dual-energy technique (d). At the iodine-free position, relative errors are almost $0 \%$ for the entire region inside the phantom for both single-energy and dual-energy techniques ((a) and (b)).

and the corresponding attenuation map. The distribution of the measured LAC values in terms of dispersion was much broader with the single-energy technique than with the dual-energy technique when the attenuation maps were obtained at the iodine position. These results suggested that, although dense materials could affect attenuation maps by producing artifacts, dual-energy CT could mitigate such artifacts, providing better attenuation maps. The authors believe that dual-energy virtual monochromatic CT can be a good option for more accurate CTAC, especially for patients who have dense materials in their bodies.

The idea of dual-energy CTAC for SPECT reconstruction was not novel; this potential use for dual-energy CT was originally proposed in the early 1990s in order to obtain an energy-corrected attenuation map for SPECT reconstruction [7]-[9]. In their work, Hasegawa et al. also reported that the dual-energy CT-based attenuation map showed minimal streak or cupping artifacts [9]. On the other hand, in 1994 LaCroix et al. described several difficulties they encountered in performing dual-energy measurements: it was time-consuming; it required an increased radiation dose; and the performance suffered from spectral overlap between low- and high-energy beams [12]. However, these technical difficulties had been overcome in recent years. The secondgeneration dual-source CT scanner used in this study could simultaneously acquire 128-slice low- and high-energy CT data at a subsecond rotation time. Spectral separation between low- and high-energy beams had been significantly improved by applying an additional tin filter to the high-energy beam [33] [34]. The tin filter was also useful in keeping the radiation dose similar to or even lower than that in single-energy CT. In fact, the CTDIvol for the dual-energy scan was lower (1.93 mGy) than that for the single-energy scan (3.43 mGy) in our phantom study. To our knowledge, the present study was the first one that used modern, commercially available dual-energy virtual monochromatic CT to improve accuracy of attenuation maps for SPECT reconstruction in the presence of dense materials.

The selection of the virtual monochromatic energy was an important issue for the application of dual-energy virtual monochromatic CT [25] [27] because not only image contrast but also the efficacy of artifact reduction depended on the energy setting. In this study, we determined the optimal energy to be $110 \mathrm{keV}$ because the artifacts were maximally reduced at this energy. Although we did not have any theoretical evidence that this energy level was optimal, this energy setting was almost consistent with the setting reported in a previous paper by Bamberg et al., which described that energies around $105 \mathrm{keV}$ were robust enough to provide an optimal visualization of metallic implants [25].

In this study, we used a dual-source CT scanner to obtain dual-energy CT data. It should be noted that dual- 
energy CTAC can also be provided by other dual-energy CT scanners having a variety of source-detector geometry and/or scintillator properties, depending on the manufacturer [20] [37] [38]. For example, dual-energy CT with fast-kVp switching allows sinogram-based virtual monochromatic imaging, which can reduce such artifacts as well [20]. Although current commercially available hybrid SPECT/CT scanners do not implement any dualenergy CT features, it is conceivable that future SPECT/CT scanners will have this capability. From a practical standpoint, for instance, a cerebral blood flow SPECT study with dual-energy CTAC does not necessarily require a hybrid SPECT/CT scanner because of the ease of co-registration of CT and SPECT data [5] [39]. Dualenergy CT data obtained with a stand-alone CT scanner can be utilized for the SPECT CTAC procedure.

This preliminary study has a few limitations. First, our study was not performed on humans because the dual-energy CT scanner we used was located in an emergency and critical care facility. Human studies involving healthy subjects or nonemergency patients were neither practical nor acceptable in the setting of emergent care. Second, we only performed a simple phantom experiment using a pair of undiluted iodine solutions. Although we were able to reduce artifacts from the iodine solutions on the attenuation map using virtual monochromatic imaging, we have not addressed the disadvantages of this strategy. For instance, virtual monochromatic imaging might not work appropriately for dense materials themselves if their attenuation exceeds the dynamic range of the scanner. Thus, further investigations with more realistic phantom experiments are necessary. Third, we have not answered the following question: why only the portions of observed artifacts in the attenuation map that had positive error values (Figure 7(c)) propagated into the SPECT image (Figure 4(f)). Numerical simulation studies using a more general reconstruction algorithm (rather than the vendor-supplied three-dimensional iterative reconstruction algorithm) are required to better understand how this phenomenon occurred.

In conclusion, dual-energy virtual monochromatic CT can effectively reduce artifacts arising from dense materials and thereby improve the accuracy of attenuation maps. This technique will contribute to more reliable SPECT reconstruction, especially when dense materials exist in the scan field of view.

\section{Acknowledgements}

This study was supported in part by the Japan Society for the Promotion of Science (JSPS) Grants-in-Aid for Scientific Research, “KAKENHI” (No. 25460751 and No. 26350471). The authors gratefully acknowledge the assistance of the staff at the Trauma and Acute Critical Care Center (TACCC) of Osaka University Hospital, especially Mr. Takeo Azuma, senior radiological technologist.

\section{Declaration of Interest}

The authors declare no conflicts of interest with respect to this paper.

\section{References}

[1] Pazhenkottil, A.P., Ghadri, J.R., Nkoulou, R.N., Wolfrum, M., Buechel, R.R., Kuest, S.M., Husmann, L., Herzog, B.A., Gaemperli, O. and Kaufmann, P.A. (2011) Improved Outcome Prediction by SPECT Myocardial Perfusion Imaging After CT Attenuation Correction. Journal of Nuclear Medicine, 52, 196-200. http://dx.doi.org/10.2967/jnumed.110.080580

[2] Stodilka, R.Z., Kemp, B.J., Prato, F.S. and Nicholson, R.L. (1998) Importance of Bone Attenuation in Brain SPECT Quantification. Journal of Nuclear Medicine, 39, 190-197.

[3] Zaidi, H. and Hasegawa, B. (2003) Determination of the Attenuation Map in Emission Tomography. Journal of Nuclear Medicine, 44, 291-315.

[4] Chang, L.T. (1973) A Method for Attenuation Correction in Radionuclide Computed Tomography. IEEE Transactions on Nuclear Science, 25, 638-643. http://dx.doi.org/10.1109/TNS.1978.4329385

[5] Hayashi, M., Deguchi, J., Utsunomiya, K., Yamada, M., Komori, T., Takeuchi, M., Kanna, K. and Narabayashi, I. (2005) Comparison of Methods of Attenuation and Scatter Correction in Brain Perfusion SPECT. Journal of Nuclear Medicine Technology, 33, 224-229.

[6] Shimosegawa, E., Fujino, K., Kato, H. and Hatazawa, J. (2013) Quantitative CBF Measurement Using an Integrated SPECT/CT System: Validation of Three-Dimensional Ordered-Subset Expectation Maximization and CT-Based Attenuation Correction by Comparing with O-15 Water PET. Annals of Nuclear Medicine, 27, 822-833. http://dx.doi.org/10.1007/s12149-013-0752-2

[7] Lang, T.F., Hasegawa, B.H., Liew, S.C., Brown, J.K., Blankespoor, S., Reilly, S.M., Gingold, E.L. and Cann, C.E. 
(1991) A Prototype Emission-Transmission Imaging System. Conference Record of the 1991 IEEE Nuclear Science Symposium and Medical Imaging Conference, 3, 1902-1906. http://dx.doi.org/10.1109/NSSMIC.1991.259246

[8] Lang, T.F., Hasegawa, B.H., Liew, S.C., Brown, J.K., Blankespoor, S.C., Reilly, S.M., Gingold, E.L. and Cann, C.E. (1992) Description of a Prototype Emission-Transmission Computed Tomography Imaging System. Journal of Nuclear Medicine, 33, 1881-1887.

[9] Hasegawa, B.H., Lang, T.F., Brown, J.K., Gingold, E.L. and Blankespoor, S.C. (1992) SPECT Reconstruction Using Uniform and Object-Specific Attenuation Maps with Emission-Transmission CT. IEEE Conference on Nuclear Science Symposium and Medical Imaging, 2, 1059-1061. http://dx.doi.org/10.1109/NSSMIC.1992.301475

[10] Kalki, K., Brown, J.K., Blankespoor, S.C., Hasegawa, B.H., Dae, M.W., Chin, M. and Stillson, C.A. (1996) Myocardial Perfusion Imaging with a Correlated X-Ray CT and SPECT System: An Animal Study. IEEE Transactions on Nuclear Science, 43, 2000-2007. http://dx.doi.org/10.1109/23.507260

[11] Kalki, K., Blankespoor, S.C., Brown, J.K., Hasegawa, B.H., Dae, M.W., Chin, M. and Stillson, C.A. (1997) Myocardial Perfusion Imaging with a Combined X-Ray CT and SPECT System. Journal of Nuclear Medicine, 38, 1535-1540.

[12] LaCroix, K.J., Tsui, B.M.W., Hasegawa, B.H. and Brown, J.K. (1994) Investigation of the Use of X-Ray CT Images for Attenuation Compensation in SPECT. IEEE Transactions on Nuclear Science, 41, 2793-2799. http://dx.doi.org/10.1109/23.340649

[13] Fleming, J.S. (1989) A Technique for Using CT Images in Attenuation Correction and Quantification in SPECT. Nuclear Medicine Communications, 10, 83-97. http://dx.doi.org/10.1097/00006231-198902000-00002

[14] Burger, C., Goerres, G., Schoenes, S., Buck, A., Lonn, A.H. and von Schulthess, G.K. (2002) PET Attenuation Coefficients from CT Images: Experimental Evaluation of the Transformation of CT into PET 511-keV Attenuation Coefficients. European Journal of Nuclear Medicine and Molecular Imaging, 29, 922-927. http://dx.doi.org/10.1007/s00259-002-0796-3

[15] Kinahan, P.E., Townsend, D.W., Beyer, T. and Sashin, D. (1998) Attenuation Correction for a Combined 3D PET/CT Scanner. Medical Physics, 25, 2046-2053. http://dx.doi.org/10.1118/1.598392

[16] von Schulthess, G.K., Steinert, H.C. and Hany, T.F. (2006) Integrated PET/CT: Current Applications and Future Directions. Radiology, 238, 405-422. http://dx.doi.org/10.1148/radiol.2382041977

[17] Mariani, G., Bruselli, L., Kuwert, T., Kim, E.E., Flotats, A., Israel, O., Dondi, M. and Watanabe, N. (2010) A Review on the Clinical Uses of SPECT/CT. European Journal of Nuclear Medicine and Molecular Imaging, 37, 1959-1985. http://dx.doi.org/10.1007/s00259-010-1390-8

[18] Ishii, K., Hanaoka, K., Okada, M., Kumano, S., Komeya, Y., Tsuchiya, N., Hosono, M. and Murakami, T. (2012) Impact of CT Attenuation Correction by SPECT/CT in Brain Perfusion Images. Annals of Nuclear Medicine, 26, 241-247. http://dx.doi.org/10.1007/s12149-011-0567-y

[19] Barrett, J.F. and Keat, N. (2004) Artifacts in CT: Recognition and Avoidance. RadioGraphics, 24, 1679-1691. http://dx.doi.org/10.1148/rg.246045065

[20] Pessis, E., Campagna, R., Sverzut, J.M., Bach, F., Rodallec, M., Guerini, H., Feydy, A. and Drapé J.L. (2013) Virtual Monochromatic Spectral Imaging with Fast Kilovoltage Switching: Reduction of Metal Artifacts at CT. RadioGraphics, 33, 573-583. http://dx.doi.org/10.1148/rg.332125124

[21] Brooks, R.A. and Di Chiro, G. (1976) Beam Hardening in X-Ray Reconstructive Tomography. Physics in Medicine and Biology, 21, 390-398. http://dx.doi.org/10.1088/0031-9155/21/3/004

[22] Suzuki, A., Koshida, K. and Matsubara, K. (2013) Adjustment of Overestimated CT-Based Attenuation Correction on Bone SPECT/CT after Hip-Resurfacing Arthroplasty. Journal of Nuclear Medicine Technology, 41, 203-207. http://dx.doi.org/10.2967/jnmt.113.121152

[23] Bonta, D.V. and Wahl, R.L. (2010) Overcorrection of Iodinated Contrast Attenuation in SPECT-CT: Phantom Studies. Medical Physics, 37, 4897-4901. http://dx.doi.org/10.1118/1.3483100

[24] Alvarez, R.E. and Macovski, A. (1976) Energy-Selective Reconstructions in X-Ray Computerized Tomography. Physics in Medicine and Biology, 21, 733-744. http://dx.doi.org/10.1088/0031-9155/21/5/002

[25] Bamberg, F., Dierks, A., Nikolaou, K., Reiser, M.F., Becker, C.R. and Johnson, T.R. (2011) Metal Artifact Reduction by Dual-Energy Computed Tomography Using Monoenergetic Extrapolation. European Radiology, 21, 1424-1429. http://dx.doi.org/10.1007/s00330-011-2062-1

[26] Meinel, F.G., Bischoff, B., Zhang, Q., Bamberg, F., Reiser, M.F. and Johnson, T.R. (2012) Metal Artifact Reduction by Dual-Energy Computed Tomography Using Energetic Extrapolation: A Systematically Optimized Protocol. Iinvestigative Radiology, 47, 406-414. http://dx.doi.org/10.1097/RLI.0b013e31824c86a3

[27] Zhou, C., Zhao, Y.E., Luo, S., Shi, H., Li, L., Zheng, L., Zhang, L.J. and Lu, G. (2011) Monoenergetic Imaging of Dual-Energy CT Reduces Artifacts from Implanted Metal Orthopedic Devices in Patients with Fractures. Academic 
Radiology, 18, 1252-1257. http://dx.doi.org/10.1016/j.acra.2011.05.009

[28] Kalender, W.A., Perman, W.H., Vetter, J.R. and Klotz, E. (1986) Evaluation of a Prototype Dual-Energy Computed Tomographic Apparatus. I. Phantom Studies. Medical Physics, 13, 334-339. http://dx.doi.org/10.1118/1.595958

[29] Guy, M.J., Castellano-Smith, I.A., Flower, M.A., Flux, G.D., Ott, R.J. and Visvikis, D. (1998) DETECT-Dual Energy Transmission Estimation CT-For Improved Attenuation Correction in SPECT and PET. IEEE Transactions on Nuclear Science, 45, 1261-1267. http://dx.doi.org/10.1109/23.682013

[30] Kinahan, P.E., Fessler, J.A., Alessio, A.M. and Lewellen, T.K. (2004) Quantitative Attenuation Correction for PET/CT Using Iterative Reconstruction of Low-Dose Dual-Energy CT. 2004 IEEE Nuclear Science Symposium Conference Record, 5, 3285-3289. http://dx.doi.org/10.1109/NSSMIC.2004.1466391

[31] Rehfeld, N.S., Heismann, B.J., Kupferschlager, J., Aschoff, P., Christ, G., Pfannenberg, A.C. and Pichler, B.J. (2008) Single and Dual Energy Attenuation Correction in PET/CT in the Presence of Iodine-Based Contrast Agents. Medical Physics, 35, 1959-1969. http://dx.doi.org/10.1118/1.2903476

[32] Huh, W., Fessler, J.A., Alessio, A.M. and Kinahan, P.E. (2009) Fast kVp-Switching Dual-Energy CT for PET Attenuation Correction. 2009 IEEE Nuclear Science Symposium Conference Record (NSS/MIC), Orlando, 24 October-1 November 2009, 2510-2515.

[33] Primak, A.N., Ramirez Giraldo, J.C., Liu, X., Yu, L. and McCollough, C.H. (2009) Improved Dual-Energy Material Discrimination for Dual-Source CT by Means of Additional Spectral Filtration. Medical Physics, 36, 1359-1369. http://dx.doi.org/10.1118/1.3083567

[34] Primak, A.N., Giraldo, J.C., Eusemann, C.D., Schmidt, B., Kantor, B., Fletcher, J.G. and McCollough, C.H. (2010) Dual-Source Dual-Energy CT with Additional Tin Filtration: Dose and Image Quality Evaluation in Phantoms and In Vivo. American Journal of Roentgenology, 195, 1164-1174. http://dx.doi.org/10.2214/AJR.09.3956

[35] Vija, A.H., Hawman, E.G. and Engdahl, J.C. (2004) Analysis of a SPECT OSEM Reconstruction Method with 3D Beam Modeling and Optional Attenuation Correction: Phantom Studies. 2003 IEEE Nuclear Science Symposium Conference Record, 4, 2662-2666.

[36] Zeintl, J., Vija, A.H., Yahil, A., Hornegger, J. and Kuwert, T. (2010) Quantitative Accuracy of Clinical ${ }^{99 \mathrm{~m}}$ Tc SPECT/ CT Using Ordered-Subset Expectation Maximization with Three-Dimensional Resolution Recovery, Attenuation, and Scatter Correction. Journal of Nuclear Medicine, 51, 921-928. http://dx.doi.org/10.2967/jnumed.109.071571

[37] Flohr, T.G., McCollough, C.H., Bruder, H., Petersilka, M., Gruber, K., Suss, C., Grasruck, M., Stierstorfer, K., Krauss, B., Raupach, R., Primak, A.N., Küttner, A., Achenbach, S., Becker, C., Kopp, A. and Ohnesorge, B.M. (2006) First Performance Evaluation of a Dual-Source CT (DSCT) System. European Radiology, 16, 256-268. http://dx.doi.org/10.1007/s00330-005-2919-2

[38] Boll, D.T., Merkle, E.M., Paulson, E.K. and Fleiter, T.R. (2008) Coronary Stent Patency: Dual-Energy Multidetector CT Assessment in a Pilot Study with Anthropomorphic Phantom. Radiology, 247, 687-695. http://dx.doi.org/10.1148/radiol.2473070849

[39] Ardekani, B.A., Braun, M., Hutton, B.F., Kanno, I. and Iida, H. (1995) A Fully Automatic Multimodality Image Registration Algorithm. Journal of Computer Assisted Tomography, 19, 615-623. http://dx.doi.org/10.1097/00004728-199507000-00022 